\title{
Experimental Study on the Spray Characteristics of Shear Coaxial/Swirl Coaxial Injectors Manufactured by SLM Process
}

\author{
JongHyeon Ahn¹, Cheolwoong Kang1, Dain Lee ${ }^{1}$, Gijeong Jeong ${ }^{1}$, Kyubok Ahn¹, \\ Ha Young Lim² \\ ${ }^{1}$ School of Mechanical Engineering, Chungbuk National University, Cheongju, Republic of \\ Korea \\ 2Launcher Propulsion Control Team, Korea Aerospace Research Institute, Daejeon, \\ Republic of Korea \\ *Corresponding author email : kbahn@cbnu.ac.kr
}

\begin{abstract}
A 3D printing shear/swirl coaxial injector was made to apply a 1-ton methane engine. The shear coaxial type was manufactured with a kind of recess length of $5.5 \mathrm{~mm}$, and the swirl coaxial type was produced with four types of recess lengths of $0,2,4$, and $5.5 \mathrm{~mm}$. Results of cold-flow test conducted using water and air as simulated propellants at room temperature and pressure. Differences in spray patterns appeared depending on the internal structure of the injector and the recess length. Further, in the swirl coaxial injector, a spray vibration phenomenon accompanied by high-frequency noise arose, and to investigate this, dynamic pressure measurement was performed. Dynamic pressure data was analysed through FFT(Fast Fourier Transform), and it was determined whether or not self-pulsation occurred from the PSD(Power Spectral Density) consequence. As a result, it can be confirmed that the swirl coaxial injector's recess length is an essential parameter for self-pulsation. Furthermore, based on the perturbation results, self-pulsation occurs through the two propellants' interaction and propagates from the gas side to the liquid side.
\end{abstract}

\section{Keywords}

Self-Pulsation, Shear Coaxial Injector, Spray Characteristic, Swirl Coaxial Injector

\section{Introduction}

Shear coaxial injectors and swirl coaxial injectors are widely used in liquid rocket engines using a liquid/gas propellant combination. In the case of a shear coaxial injector, a liquid oxidizer is injected into the center of the injector at a relatively slow velocity, and gaseous fuel at high velocity is injected around it [1]. In a swirl coaxial injector, the internal oxidizer inlet is machined into a tangential hole. The liquid oxidizer is injected in a swirl motion and interacts with the gaseous fuel injected around it [2]. These coaxial injectors are significantly affected by atomization and mixing performance according to the injector recess length $\left(L_{R}\right)[3,4]$. However, when designing a swirl coaxial injector, it is necessary to consider the self-pulsation phenomenon when determining the recess length. This self-pulsation phenomenon was first discovered by Bazarov in 1976 [5], and in subsequent studies [6], confirmed that the recess length of the injector is an essential parameter of the self-pulsation phenomenon. Since then, the self-pulsation phenomenon has been studied until now. Im et al. found that the selfpulsation phenomenon occurs due to unstable waves of the liquid sheet, and the corresponding frequencies affect the dominant wave of the liquid sheet [7]. Kang et al. argued that the breakup length of the spray shortens when the self-pulsation phenomenon occurs, but it negatively affects atomization [8]. Bai et al. showed that self-pulsation occurs due to the interaction between gas and liquid at the recess wall of the swirl sprayer. It was confirmed that the liquid with the velocity in the tangential direction collides with the wall of the recess and 
blocks the gas flow, thereby increasing the pressure at the gas post, pushing the liquid back, and decreasing the pressure, and the cycle of self-pulsation proceeds [9].

Self-pulsation occurred in the cold-flow test of coaxial injectors designed to apply a 1-ton class methane engine. Such a phenomenon may cause combustion instability during actual combustion and may further damage the engine. Therefore, this research team experimented in a room pressure environment to investigate the occurrence region of the self-pulsation phenomenon and its characteristics in advance in the experimental stage.

\section{Experimental Methods}

The injector used in the experiment was designed to apply a 1-ton thrust methane engine and was manufactured with a metal 3D printer. The propellant supplied to the injector is liquid oxygen and gaseous methane, and accordingly, the injector type is determined to be a shear/swirl coaxial type widely used in a liquid/gas propellant combination. The cross-section of each injector is shown in Fig 1. Each injector was made of 5 kinds with $L_{R}$ and internal structure as variables, and their parameter is described in Table 1.

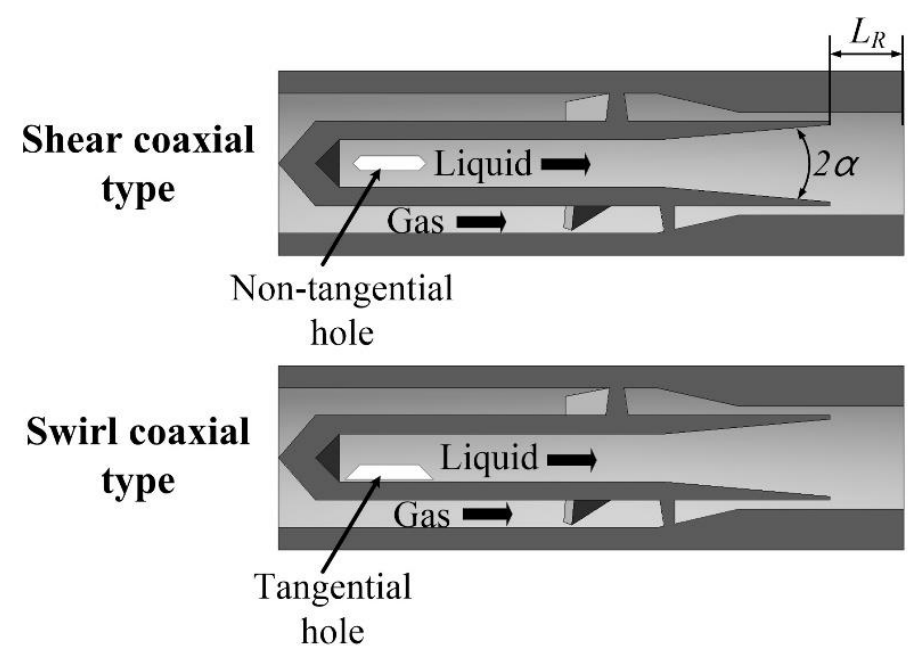

Figure 1. Closs-section of each 3D printed injector used in the experiment

Table 1 - Geometric parameters of injectors

\begin{tabular}{c|cccc}
\hline No. & O post & F post & $L_{R}$ & $2 \alpha$ \\
\hline D-12 & Straight & Straight & 5.5 & $7^{\circ}$ \\
E-0 & Swirl & Swirl & 0 & $7^{\circ}$ \\
E-2 & Swirl & Swirl & 2 & $7^{\circ}$ \\
E-4 & Swirl & Swirl & 4 & $7^{\circ}$ \\
E-5.5 & Swirl & Swirl & 5.5 & $7^{\circ}$
\end{tabular}

The schematic diagram of the experimental device is shown in Fig. 2. The cold-flow test was conducted at room temperature and pressure using water and air as simulation propellants. The water mass flow rate was controlled using a needle valve and a mass flow meter (kometer, KMS-2000, uncertainty $\pm 0.10 \%$ ) after constant pressurization of the water tank employing a high-pressure air tank and a regulator. Air was injected at a regular mass flow rate using an MFC controller after maintaining a steady pressure at the MFC (MKP, VIC-D240, uncertainty $\pm 1.0 \%$ ) inlet using a high-pressure air tank and regulator. Dynamic pressure data were 
obtained through a PCB dynamic pressure sensor (PCB, 102A14/102B, uncertainty $\pm 15.0 \% / \pm$ $10 \%$ ) installed on each propellant manifold and collected on a $\mathrm{PC}$ at a sampling rate of $20000 \mathrm{~Hz}$ through a PCB Signal Conditioner (PCB, 482A16) and NI-cDAQ. The spray image was taken with a backlight image at a sampling rate of $1000 \mathrm{~Hz}$ by placing a light source and a high-speed camera in front and behind the test stand.

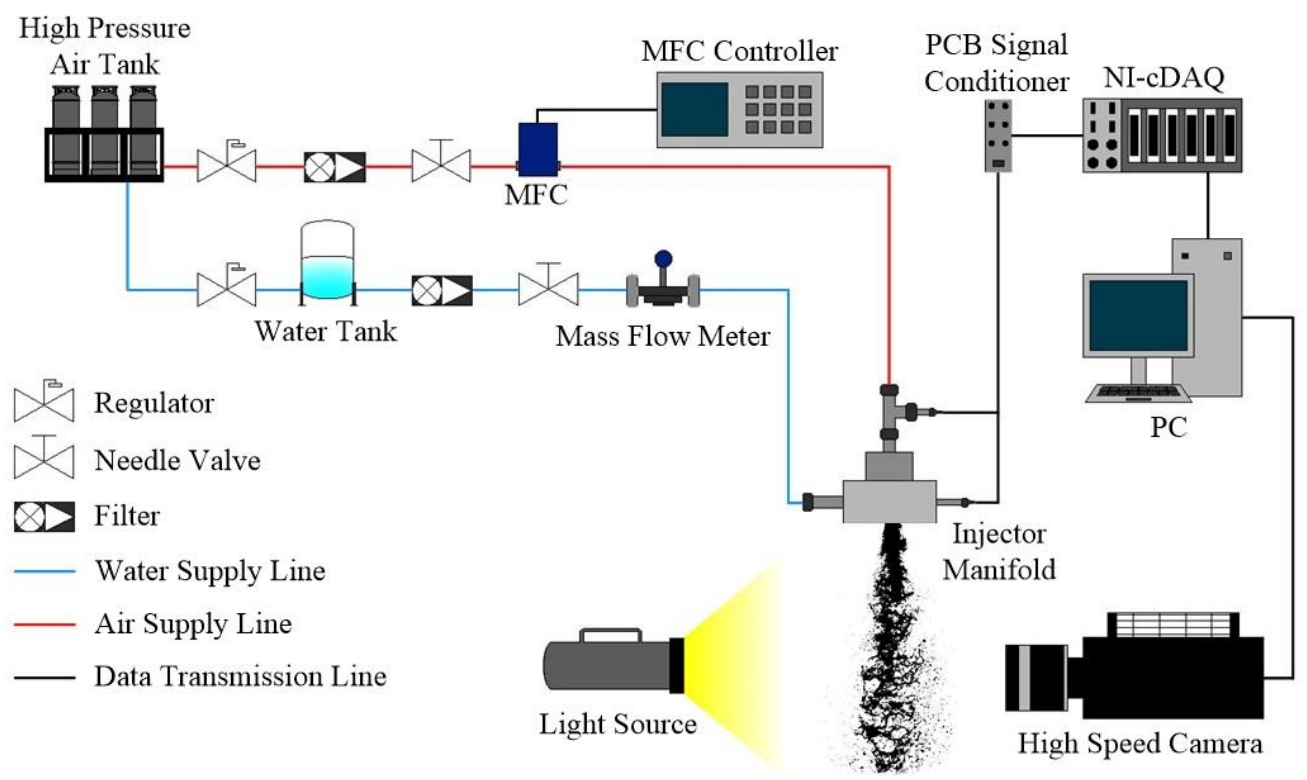

Figure 2. Schematic diagram of the experimental setup

The combustion chamber's design pressure of the methane engine to be applied is 35 bar, and the mass flow rate of a single injector at a mixing ratio of 3.0 is $121 \mathrm{~g} / \mathrm{s}$ of liquid oxygen and $40.6 \mathrm{~g} / \mathrm{s}$ of gaseous methane. This study's experimental nominal flow rate was obtained by equating the actual propellant velocity with the cold-flow test's flow velocity for simulation of the combustion environment. Besides, to investigate the self-pulsation phenomenon's occurrence and characteristics, the experimental condition was set in an extensive and dense range. The experimental conditions are shown in Table 2, and the experiment was conducted with $\dot{m}_{g} / \dot{m}_{g d}$ adjusted to $0 \sim 200 \%$ in $10 \%$ increments, and $\dot{m}_{l} / \dot{m}_{l d}$ increased from 5 to $150 \%$ in $5 \%$ intervals.

Table 2 - Nominal mass flow rate and experimental conditions for cold-flow test

\begin{tabular}{c|cc}
\hline Simulant & $\dot{m}_{d}\left[\mathrm{~g} \mathrm{~s}^{-1}\right]$ & $\dot{m} / \dot{m}_{d}[\%]$ \\
\hline Air & 2.083 & $0 \sim 200$ \\
Water & 105.170 & $5 \sim 150$
\end{tabular}

\section{Results and Discussion}

The image results of a single liquid injection and a bi-injection for each injector are shown in Fig. 3. The liquid mass flow rate is $50 \%$ of the nominal conditions, and the air mass flow rate during the bi-injection is the nominal mass flow rate. When checking the single injection results, it is confirmed that the Inj D-12 of the shear coaxial type has the oxidizer inlet hole processed to the center of the injector so that the liquid has only the axial velocity. However, swirl coaxial injectors have been found to be sprayed in swirl motion. Also, as the recess length is increased, it collides with the recess's wall due to the velocity in the tangential direction, and the 
momentum is lost, and the spray angle is further reduced. The bi-injection results show that the mixing performance has a positive effect as the recess length increases.

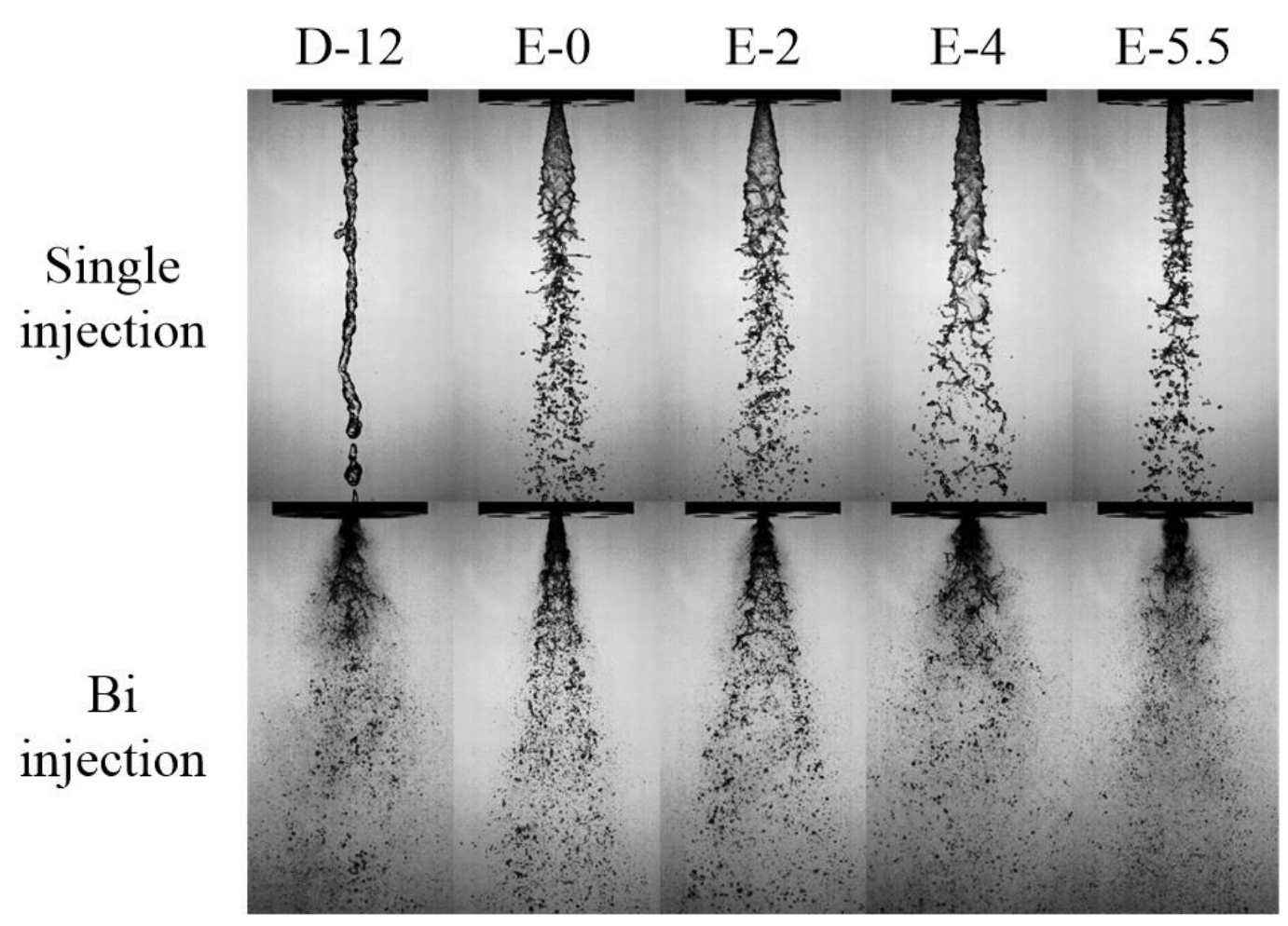

Figure 3. Instantaneous image result of each injector during single injection and bi-injection

The data of each experimental condition measured through the dynamic pressure sensor were Fast Fourier Transform analyzed. Accordingly, in the PSD result, the definition of the occurrence of self-pulsation had to proceed first. Thus, the self-pulsation is defined as shown in Fig. 4. Among the data measured in the fuel or oxidizer manifold, it was determined that self-pulsation occurred when a narrow and sharp peak appeared at a particular frequency and that no self-pulsation occurred when the peak was broad or none. Fig. 5 shows the occurrence area for each injector under all experimental conditions. In the shear coaxial type Inj D-12, self-pulsation did not appear in all experimental conditions, so it was excluded from the graph. The recess length seems to have a significant impact on the generation of self-pulsation. The increase in recess length extended the self-pulsation area but instead decreased as $L_{R}$ increased to more than $4 \mathrm{~mm}$.

Figure 6 shows the measured RMS value of the perturbation, and through this, the intensity of the self-pulsation was examined. The increase in the Reynolds number of each propellent showed a different trend. The self-pulsation intensity dependently increased on $R_{g}$, but for $R e_{l}$, it was the largest at a specific value. The magnitude of the highest RMS value found at a particular Reynolds number was larger with longer recess lengths but lower than Inj E-4 in the results of Inj E-5.5 with the most extended recess lengths.

The mass flow rate of each propellant also affected the high-frequency noise accompanying self-pulsation. Fig. 7 shows the measured peak frequencies according to the Reynolds number

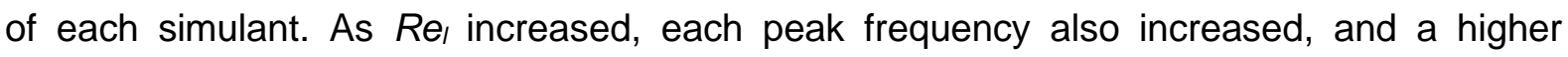
frequency perturbation occurred at a shorter recess length. The effect of the gas Reynolds number was different from that of the liquid. For Inj E-0 and E-2 with relatively short recess 
lengths, the peak frequency increased according to $R e_{g}$, but in the case of Inj E-4 and E-5.5, the peak frequency increased and converged at a specific frequency.
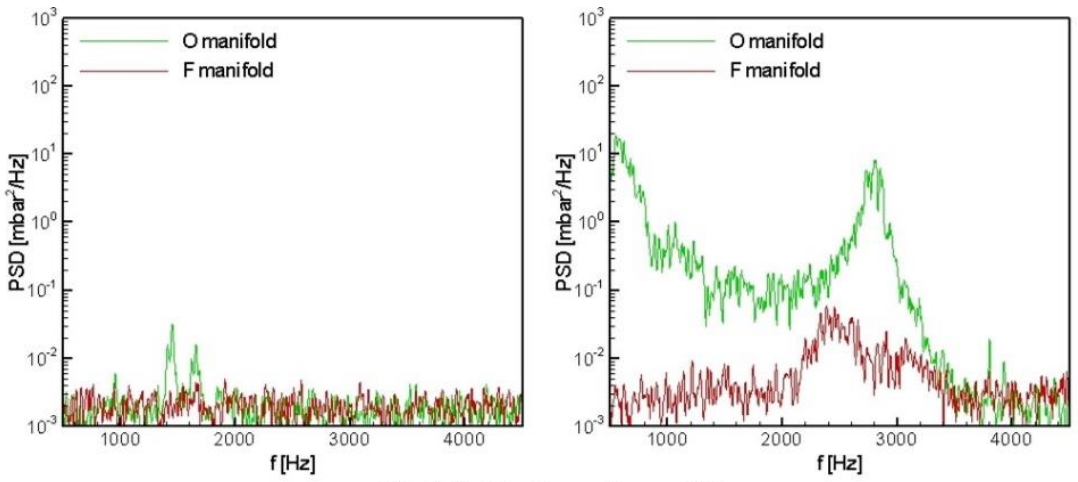

Self-Pulsation X
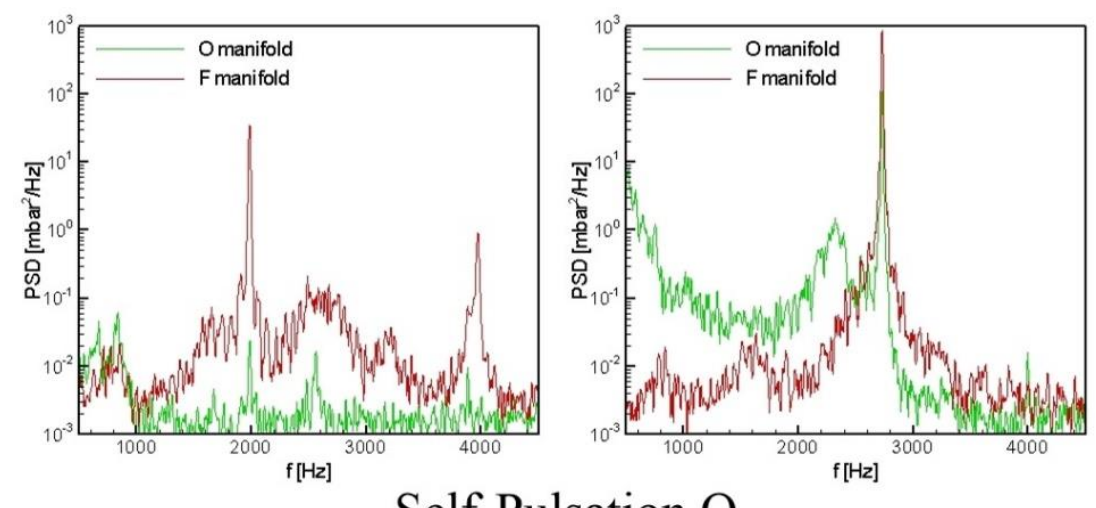

Self-Pulsation O

Figure 4. Definition of self-pulsation occurrence through power spectral density results
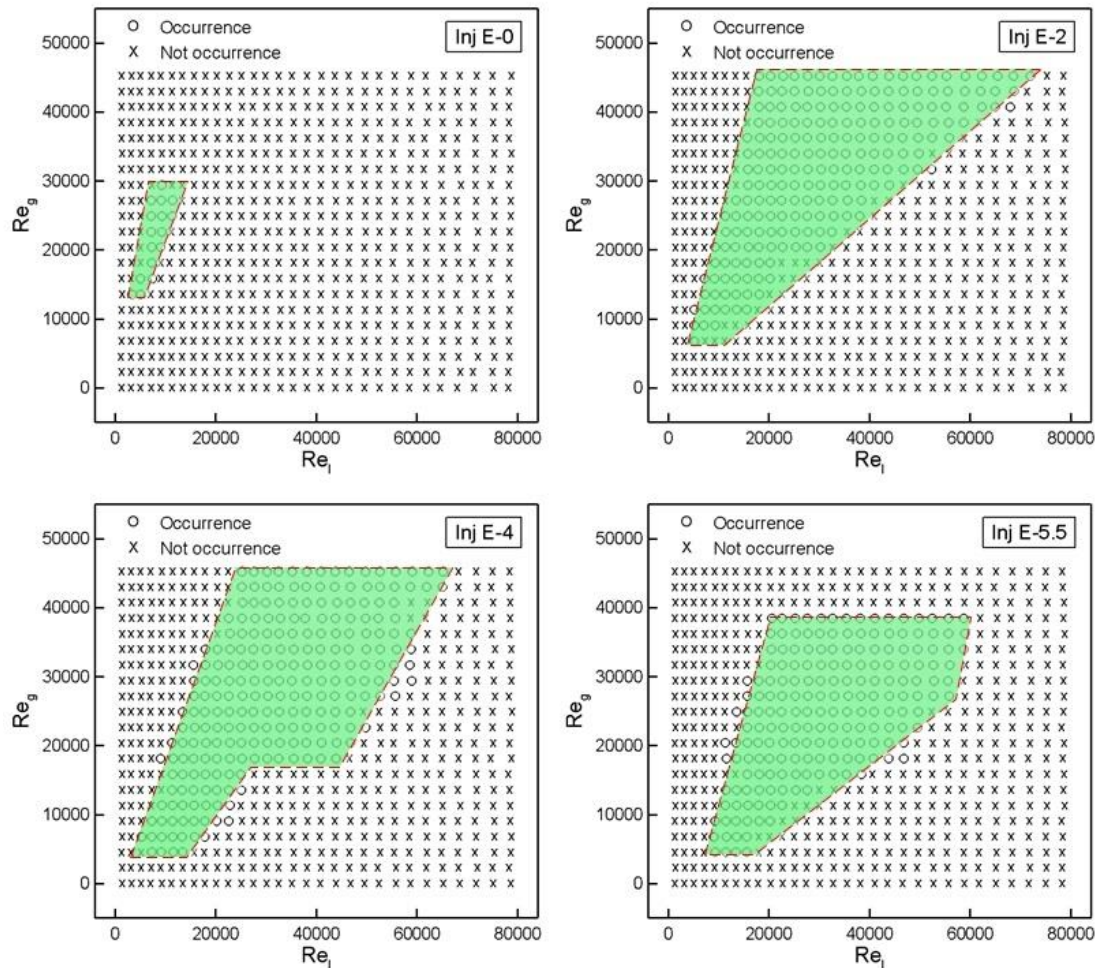

Figure 5. Self-pulsation occurrence area under experimental conditions for each injector 

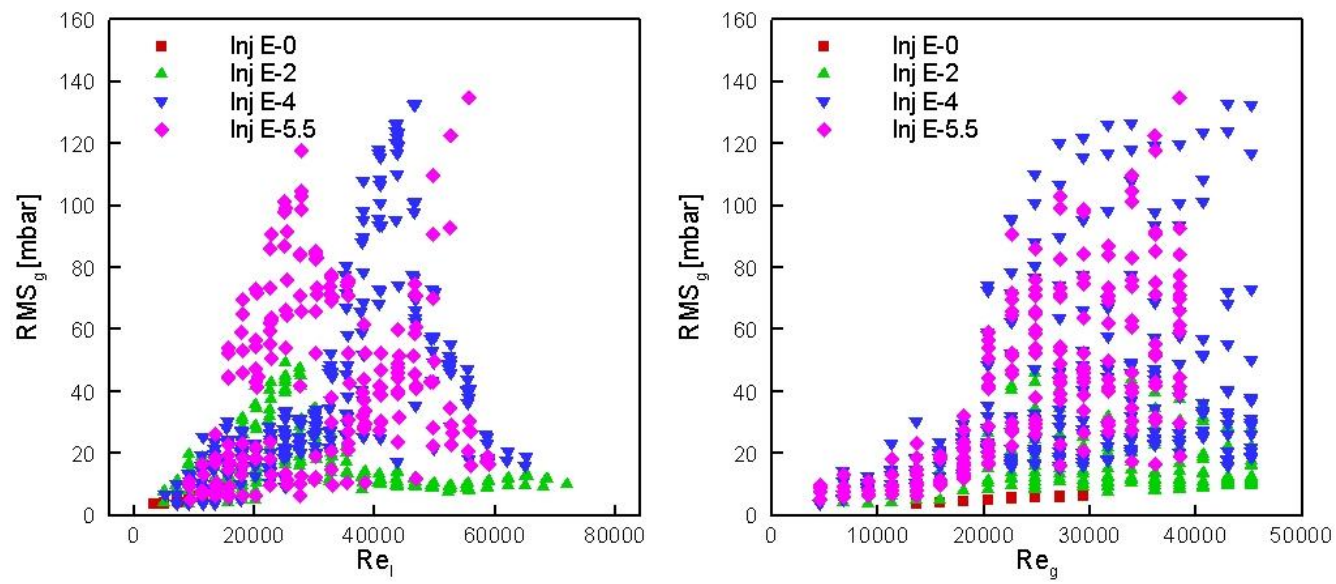

Figure 6. RMS value of perturbation for the Reynolds number of liquid (left) and gas (right) when self-pulsation occurs
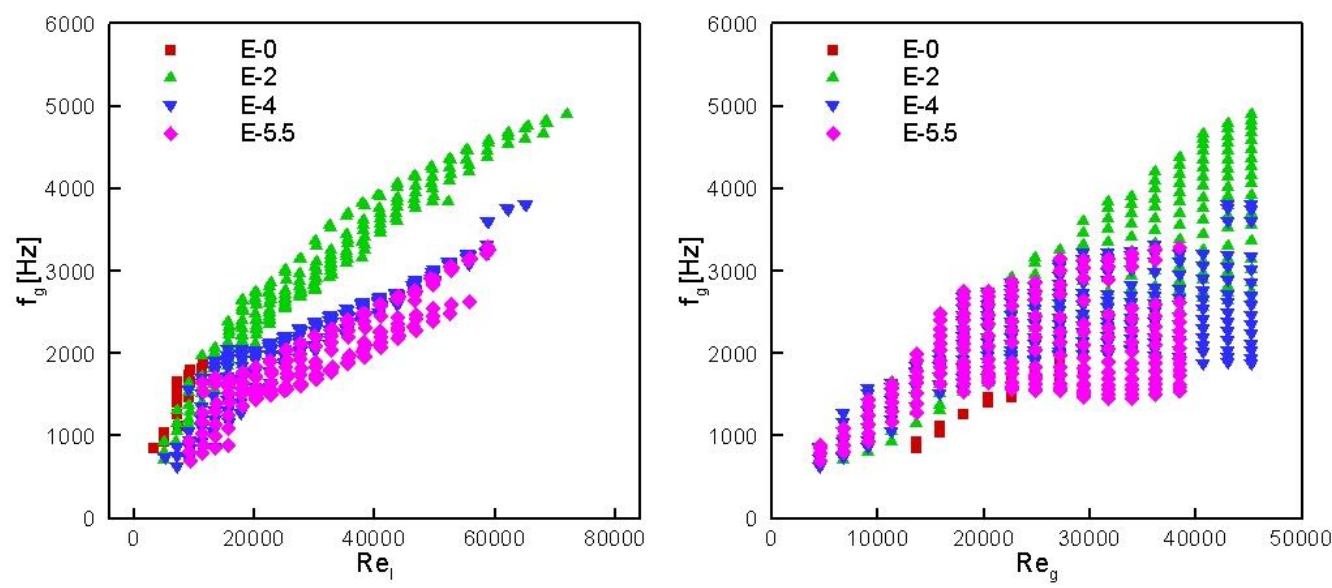

Figure 7. Perturbation peak frequency result according to Reynolds number of liquid (left) and gas (right) when self-pulsation occurs

A narrow, sharp peak indicating the occurrence of self-pulsation was first exposed on the fuel manifold side in all results. In the oxidizer manifold, this peak was only observed under some conditions. Figure 8 shows the frequency measured in the oxidizer manifold and the frequency measured in the fuel manifold together. The frequency results measured on the oxidizer manifold show that the peak is only identified under some injector's experiment conditions with a long recess. Furthermore, when each manifold's frequency results were presented together under these conditions, they were found to be approximately the same frequency. In the case of long recess injectors, as can be seen from the spray image, internal mixing increases, and mixing performance increases. Therefore, the perturbation occurs first on the gas side, and as the liquid flow rate increases, the interaction in the recess area increases, and it seems to propagate toward the liquid. 

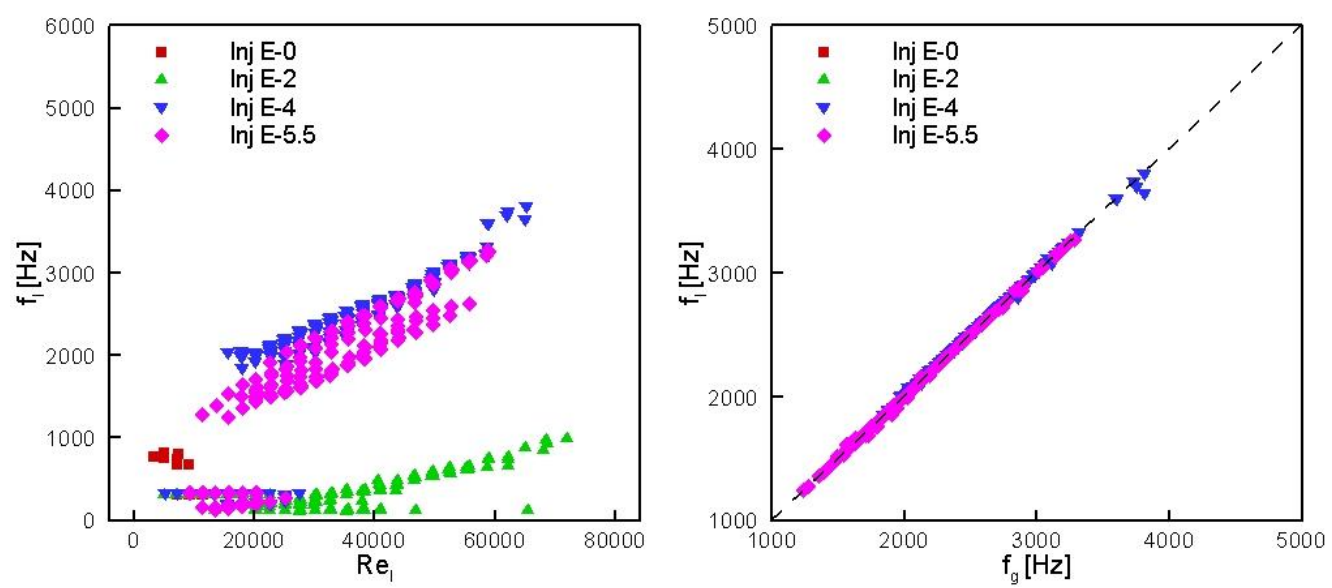

Figure 8. Peak frequency measured on the oxidizer manifold according to the liquid Reynolds number (left), and comparison of frequency measured on each manifold (right)

To check the self-pulsation frequency characteristics, the Strouhal number was calculated as shown in Equation (1).

$$
S t=f_{g} L_{R} / V
$$

Each variable used the injector recess length and the axial velocity of the swirl jet. Inj E-0, in which the self-pulsation occurrence area was relatively narrow, and the intensity was weak, were excluded. The calculated Strouhal number was a linear regression analysis using the recess length/injector outlet diameter ratio and the Reynolds number of each propellant as variables, and the empirical formula for this was as shown in Equation (2).

$$
S t=7.7287\left(L_{R} / D\right)^{0.6248} R e_{l}^{0.5529} \operatorname{Re}_{g}{ }^{-0.9078}
$$

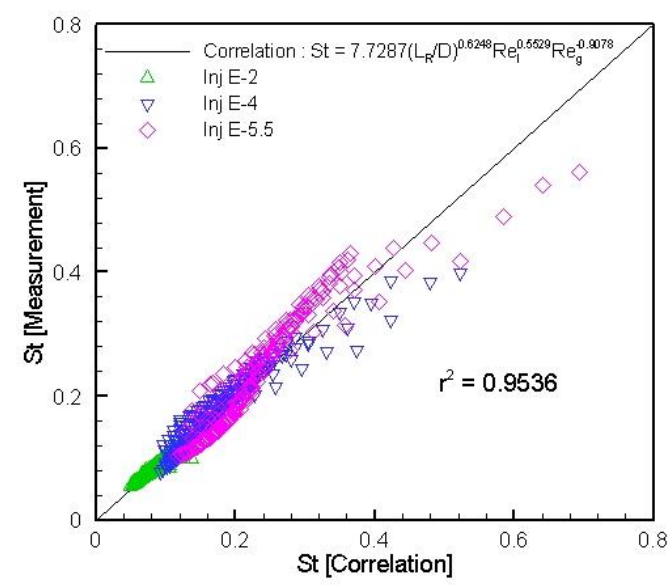

Figure 9. Results of linear regression analysis of the Strouhal number calculated through experiments

\section{Conclusions}

The injector was manufactured with a metal 3D printer to apply a 1-ton methane engine, and a self-pulsation phenomenon accompanied by high-frequency noise occurred in the cold-flow test. Accordingly, dynamic pressure measurement was performed under broad and dense experimental conditions. The self-pulsation boundary widened with the increase of $L_{R}$ but reduced in recesses longer than $4 \mathrm{~mm}$. The intensity of self-pulsation increased with the gas 
mass flow rate, but the liquid mass flow rate showed the highest point at a specific Reynolds number. The peak frequency increased with the liquid and gas Reynolds number, but in injectors with a $4 \mathrm{~mm}$ or more recess length, it rose with $R e_{g}$ and converged to a specific frequency. We also identified perturbation to the oxidizer manifold when these injectors' internal mixing became might. As a conclusion through the experiment, the recess length greatly influenced the occurrence and characteristics of self-pulsation, and to resist this, consideration of the recess length should be sufficient when designing the injector.

\section{Acknowledgments}

\section{Nomenclature}

$D \quad$ exit diameter of injector [mm]

$f_{g} \quad$ gas side frequency $\left[\mathrm{s}^{-1}\right]$

$f_{l} \quad$ liquid side frequency $\left[\mathrm{s}^{-1}\right]$

$L_{R} \quad$ recess length [mm]

$\dot{m}_{g} \quad$ air mass flow rate $\left[\mathrm{g} \mathrm{s}^{-1}\right]$

$\dot{m}_{\text {gd }} \quad$ nominal air mass flow rate $\left[\mathrm{g} \mathrm{s}^{-1}\right]$

$\dot{m}_{l} \quad$ liquid mass flow rate $\left[\mathrm{g} \mathrm{s}^{-1}\right]$

$\dot{m}_{l d} \quad$ nominal liquid mass flow rate $\left[\mathrm{g} \mathrm{s}^{-1}\right]$

$R_{g} \quad$ gas side axial Reynolds number

$R e_{\text {, }} \quad$ liquid side axial Reynolds number

St Strouhal number

$\checkmark \quad$ axial velocity $\left[\mathrm{m} \mathrm{s}^{-1}\right]$

$2 \alpha \quad$ taper angle [deg]

\section{References}

[1] Glogowski, M., and Micci, M. M., Jul. 10.-12. 1995, 31st AIAA/ASME/SAE/ASEE Joint Propulsion Conference and Exhibit

[2] Hulka, J., and Schneider, J., Jun. 28.-30. 1993, 29th AIAA/SAE/ASME/ASEE Joint Propulsion Conference and Exhibit

[3] Woodward, R. D., Pal, S., Farhangi, S., Jensen, G. E., Santoro, R. J., Jan. 8.-11. 2007, 45th AIAA Aerospace Sciences Meeting and Exhibit

[4] Yang, L., Ge, M., Zhang, M., Fu, Q., Cai, G., 2008, Journal of Propulsion and Power, 24(6), pp. 1332-1339

[5] Bazarov, V. G., and Lul'ka, L. A., 1978, Soviet Aeronautics, 3, pp. 14-18

[6] Bazarov, V. G., Jul. 10.-12. 1995, 31st AIAA/ASME/SAE/ASEE Joint Propulsion Conference and Exhibit

[7] Im, J. H., Kim, D., Han, P., Yoon, Y., Bazarov, V. G., 2009, Atomization and Sprays, 19(1), pp. $57-74$

[8] Kang, Z., Li, Q., Cheng, P., Zhang, X., Wang, Z., 2016, Acta Astronautica, 127, pp. 249259

[9] Bai, X., Li, Q., Cheng, P., Sheng, L., Kang, Z., 2018, Acta Astronautica, 151, pp. 511-521 